\title{
NATURAL HOMOMORPHISMS IN BANACH SPACES
}

\section{J. DIEUDONNE}

1. Introduction. ${ }^{1}$ In a recent paper, published in this Bulletin [7], M. E. Munroe considers the following question. Let $E$ be a Banach space, $E^{*}$ its dual, $G$ a closed linear subspace of $E, \Gamma$ the subspace of $E^{*}$ orthogonal to $G$ (that is, the space consisting of the linear functionals $x^{\prime}$ on $E$ such that $x^{\prime}(x)=0$ for every $\left.x \in G\right)$; there is then a natural homomorphism $T$ of $E^{*}$ onto the dual $G^{*}$, with kernel $\Gamma$; Munroe investigates the continuity and openness of $T$ when both $E^{*}$ and $G^{*}$ are given one of the following topologies: norm, weak, weak*, bounded weak, and bounded weak* (the topologies on $E^{*}$ and $G^{*}$ being always of the same type).

The openness of $T$ for the norm topologies is proved by Krein and Smulian [5], and is an easy consequence of the Hahn-Banach extension theorem (see $[3$, p. 124, Theorem 17a]). Munroe proves the openness of $T$ for the weak* and weak topologies (Theorems 3.3 and 3.4), but he has failed to notice that both theorems are particular cases of a general result I proved some years ago $[3$, p. 116, Theorem 7] for linearly convex spaces, by the same argument Munroe uses in his proof. I shall prove in this note that $T$ is also open for the bounded weak $k^{*}$ and bounded weak topologies on $E^{*}$ and $G^{*}$; also I shall rectify an error in the first statement of Munroe's Theorem 3.5.

2. Characterization of the bounded weak* topology on $E^{*}$. For $x \in E$ and $x^{\prime} \in E^{*}$, I shall write $x^{\prime}(x)=\left\langle x, x^{\prime}\right\rangle$; for any subset $A$ of $E$ (resp. $A^{\prime}$ of $\left.E^{*}\right), A^{0}$ (resp. $A^{\prime 0}$ ) will be the subset of $E^{*}$ (resp. $E$ ) consisting of the $x^{\prime}$ such that $\left|\left\langle x, x^{\prime}\right\rangle\right| \leqq 1$ for every $x \in A$ (resp. the $x$ such that $\left|\left\langle x, x^{\prime}\right\rangle\right| \leqq 1$ for every $\left.x^{\prime} \in A^{\prime}\right)$; clearly $A^{0}$ is convex and weakly* closed, $A^{\prime 0}$ convex and closed. In the following, the word "compact" is used in the Bourbaki sense (="bicompact").

Lemma 1. For every subset $A$ of $E$, the set $A^{00}$ is the smallest convex and closed subset of $E$ containing both $A$ and $-A$.

It is clear that $A \subset A^{00}$ and $-A \subset A^{00}$; if $A_{1}$ is the smallest convex closed set containing $A$ and $-A, A_{1} \subset A^{00}$. On the other hand, let $x$ be a point in $E$ not belonging to $A_{1}$; there exists a closed hyperplane $H$

Received by the editors September 24, 1948 and, in revised form, November 8, 1948.

1 Numbers in brackets refer to the bibliography at the end of the paper. 
separating $x$ and $A_{1}$, by the Hahn-Banach theorem; this means that there is an $x^{\prime} \in E^{*}$ such that $\left\langle y, x^{\prime}\right\rangle \leqq 1$ for every $y \in A_{1}$, and $\left\langle x, x^{\prime}\right\rangle>1$; as $-A_{1}=A_{1}$, one has also $\left|\left\langle y, x^{\prime}\right\rangle\right| \leqq 1$ for every $y \in A_{1}$, and as $A \subset A_{1}$, $x^{\prime} \in A^{0}$, and therefore $x \notin A^{00}$, which proves the lemma.

\section{Lemma 2 (MAZUR). If $A$ is strongly compact in $E$, so is $A^{00}$.}

$A^{00}$ is the closure of the convex hull $B$ of $A \cup(-A)$ in $E$, and therefore is complete for the metric on $E$; it is therefore enough to prove that $B$ is strongly precompact. For every $\epsilon>0$, there exist a finite number of points $x_{i}$ in $A \cup(-A)(1 \leqq i \leqq n)$ such that any $x \in A \cup(-A)$ is contained in one of the balls $\left\|x-x_{i}\right\| \leqq \epsilon$; if $C$ is the convex hull of the finite set of the $x_{i}$, every point of $B$ is at a distance not greater than $\epsilon$ from a point of $C$. But $C$ is finite-dimensional and bounded, therefore compact; there are therefore a finite number of points $y_{j}$ of $C(1 \leqq j \leqq n)$ such that any point of $C$ is at a distance not greater than $\epsilon$ from one of the $y_{j}$; therefore every point of $B$ is at a distance not greater than $2 \epsilon$ from one of the $y_{j}$, which completes the proof.

The bounded weak* topology on $E^{*}$ may be defined as the finest topology on $E^{*}$ which, on every ball $\left\|x^{\prime}\right\| \leqq r$, coincides with the weak* topology. The open neighborhoods of the origin for that topology are therefore the sets $U$ containing the origin and such that, for every ball $S, U \cap S$ is open for the topology induced on $S$ by the weak* topology. We now prove the following theorem:

THEOREM 1. A fundamental system of neighborhoods of the origin for the bounded weak* topology consists of the sets $K^{0}$, where $K$ is an arbitrary strongly compact subset of $E$ (in other words, the bounded weak* topology is identical with the k-topology in the sense of Arens [1, p. 789]).

We shall see that this theorem is practically only a new formulation of a result of Banach [2, pp. 119-120]. Let us prove first that a set $K^{0}$ is a neighborhood of $O$ for the bounded weak* topology. Let $S$ be any ball $\left\|x^{\prime}\right\| \leqq r$; as $K$ is compact, there exist a finite number of points $x_{i}$ in $K(1 \leqq i \leqq n)$ such that any point of $K$ is at a distance not greater than $1 / 2 r$ from one of the points $x_{i}$. Let $W$ be the neighborhood of $O$ in the weak ${ }^{*}$ topology, defined by the $n$ inequalities $\left|\left\langle x_{i}, x^{\prime}\right\rangle\right| \leqq 1 / 2$; for any $x^{\prime} \in W \cap S$, and any $x \in K$, there is a point $x_{i}$ such that $\left\|x-x_{i}\right\| \leqq 1 / 2 r$, and therefore

$$
\left|\left\langle x, x^{\prime}\right\rangle\right|=\left|\left\langle x_{i}, x^{\prime}\right\rangle+\left\langle x-x_{i}, x^{\prime}\right\rangle\right| \leqq \frac{1}{2}+\frac{1}{2 r}\left\|x^{\prime}\right\| \leqq 1
$$

which proves that $W \cap S$ is contained in $K^{0}$. 
We now prove conversely that if $U$ is any open neighborhood of the origin for the bounded weak* topology, it contains a set $K^{0}$. This will be a consequence of the following lemma:

LEMMA 3. There exists in $E$ a finite set $A_{1}$ of points of norm greater than 1 and, for every $n>0$, a finite set $B_{n}$ of points of norm not greater than $1 / n$, such that, if $A_{n+1}=A_{n} \cup B_{n}$ for $n>0$, the intersection of $A_{n}^{0}$ with the ball $\left\|x^{\prime}\right\| \leqq n$ is contained in $U$.

Suppose that lemma is proved: the union $A$ of all sets $A_{n}$ and of the point $O$ is obviously strongly compact; let $S$ be any ball $\left\|x^{\prime}\right\| \leqq r$, and $n$ an integer greater than $r$; Lemma 3 shows that the intersection $S \cap A_{n}^{0}$ is contained in $U$, and a fortiori $S \cap A^{\circ} \subset U$; as the radius of $S$ is arbitrary, $A^{\circ} \subset U$.

Lemma 3 is substantially the result of Banach quoted above; for the sake of completeness, we reproduce its proof. The intersection of $U$ with the ball $\left\|x^{\prime}\right\| \leqq 1$ contains a weak* neighborhood of $O$, and therefore there exists a finite set $A_{1}$ of $E$ such that the intersection of $A_{1}^{0}$ with the ball $\left\|x^{\prime}\right\| \leqq 1$ is contained in $U$; it may obviously be supposed that the norms of the elements in $A_{1}$ are greater than 1. Now argue inductively in the following way: suppose $A_{n}$ is defined as a finite set in $E$ such that the intersection of $A_{n}^{0}$ with the ball $S_{n}:\left\|x^{\prime}\right\| \leqq n$ is contained in $U$, and let us show that there exists a finite set $B_{n}$ of points of norm $\leqq 1 / n$ such that the intersection of $\left(A_{n} \cup B_{n}\right)^{0}$ with the ball $S_{n+1}:\left\|x^{\prime}\right\| \leqq n+1$ is contained in $U$. Suppose that proposition were not true, and let $K$ be the intersection of $S_{n+1}$ with the complement of $U$ in $E^{*} ; K$ is closed for the bounded weak* topology, and therefore for the weak* topology, since on $S_{n+1}$ both topologies coincide; as $K$ is bounded, it is weakly* compact [3, p. 128, Theorem 22]. For every finite subset $B$ of the ball $\|x\| \leqq 1 / n$ there would exist a nonvoid subset $H(B)$ of $K$, contained in $\left(A_{n} \cup B\right)^{0}$; the subsets $H(B)$ constitute obviously a filter base on $K$, having therefore a cluster point $x_{0}^{\prime}$ in $K$ for the weak* topology, since $K$ is weakly* compact. For every $\epsilon>0$ and every $x \in E$ such that $\|x\| \leqq 1 / n$, there exists therefore an $x^{\prime} \in K$ such that $\left|\left\langle x, x^{\prime}\right\rangle\right| \leqq 1$ and $\left|\left\langle x, x^{\prime}-x_{0}^{\prime}\right\rangle\right| \leqq \epsilon$, whence $\left|\left\langle x, x_{0}^{\prime}\right\rangle\right| \leqq 1+\epsilon$, and as $\epsilon$ is arbitrary, $\left|\left\langle x, x_{0}^{\prime}\right\rangle\right| \leqq 1$; as that relation holds for every $x$ such that $\|x\| \leqq 1 / n$, we have $\left\|x_{0}^{\prime}\right\| \leqq n$. The same argument shows that, for every $x \in A_{n}$, one has $\left|\left\langle x, x_{0}^{\prime}\right\rangle\right| \leqq 1$, in other words $x_{0}^{\prime} \in A_{n}^{0} \cap S_{n}$, and the induction hypothesis implies $x_{0}^{\prime} \in U$, which is absurd, since $x_{0}^{\prime}$ is in $K$.

THEOREM 2. The natural homomorphism $T$ is open for the bounded weak* topology.

We shall adapt to our purpose an argument of Köthe $[4$, p. 27]. 
Let $K$ be a strongly compact subset of $E$; as $K^{00}$ is strongly compact and $\left(K^{00}\right)^{0}=K^{0}$, we may suppose that $K$ is convex and symmetric by Lemma 2. Let $U$ be the neighborhood of $O$ in $E^{*}$ for the bounded weak* topology, consisting of the elements $x^{\prime}$ such that $\left|\left\langle x, x^{\prime}\right\rangle\right|<1$ for every $x \in K$ (Theorem 1); we want to prove that $T(U)$ contains a neighborhood of the origin in $G^{*}$ for the bounded weak* topology. We shall reach that goal by proving that $T(U)$ contains all elements

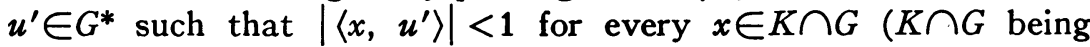
strongly compact in $G$ ). As $u^{\prime}$ may be considered as the restriction to $G$ of a linear functional $x_{0}^{\prime}$ defined in $E$, we have to prove that if $x_{0}^{\prime}$ is such that $\left|\left\langle x, x_{0}^{\prime}\right\rangle\right|<1$ for every $x \in K \cap G$, there exists a linear functional $y_{0}^{\prime}$ such that $x_{0}^{\prime}-y_{0}^{\prime} \in \Gamma$ and that $\left|\left\langle y, y_{0}^{\prime}\right\rangle\right|<1$ for every $y \in K$.

Consider in $E^{*}$ the closed subspace $H$ orthogonal to all elements of $K$, and let $\phi$ be the natural homomorphism of $E^{*}$ onto $E^{*} / H$; for every element $\phi\left(x^{\prime}\right)$ of $E^{*} / H,\left\|\phi\left(x^{\prime}\right)\right\|=\sup _{x \in K}\left|\left\langle x, x^{\prime}\right\rangle\right|$ is a norm on $E^{*} / H$, as is readily verified. Moreover, if $f$ is any linear functional on $E^{*} / H$, continuous for the topology defined by that norm, fo $\phi$ is a linear functional on $E^{*}$, such that $\left|f\left(\phi\left(x^{\prime}\right)\right)\right|$ is bounded on $K^{0}$; as $K$ is strongly compact in $E$, it is also weakly compact, and therefore, by Arens's theorem [1, p. 790, Theorem 2], there exists an element $z \in E$ such that $f\left(\phi\left(x^{\prime}\right)\right)=\left\langle z, x^{\prime}\right\rangle$ identically.

Let $d$ be the distance of $\phi\left(x_{0}^{\prime}\right)$ to the linear subspace $\phi(\Gamma)$ in the normed space $E^{*} / H$ and suppose first $\phi(\Gamma)$ is not everywhere dense in $E^{*} / H$. The Hahn-Banach extension theorem proves the existence of a continuous linear functional $f_{0}$ on $E^{*} / H$, having a norm equal to 1 , equal to 0 in the subspace $\phi(\Gamma)$, and such that $f_{0}\left(\phi\left(x_{0}^{\prime}\right)\right)=d$. Let $x_{0}$ be an element of $E$ such that $f_{0}\left(\phi\left(x^{\prime}\right)\right)=\left\langle x_{0}, x^{\prime}\right\rangle$ identically; the definition of $f_{0}$ shows that $x_{0} \in G$, and that, for every $x^{\prime} \in E^{*},\left|\left\langle x_{0}, x^{\prime}\right\rangle\right|$ $\leqq \sup _{x \in K}\left|\left\langle x, x^{\prime}\right\rangle\right|$; in particular, if $x^{\prime} \in K^{0}$, one has $\left|\left\langle x_{0}, x^{\prime}\right\rangle\right| \leqq 1$ by definition, and therefore $x_{0} \in K^{00}=K$; we thus see that $x_{0} \in K \cap G$. Finally, the definition of the distance of a point to a set in a metric space shows that, given any $\epsilon>0$, there exists $z^{\prime} \in \Gamma$ such that $\sup _{x \in K}\left|\left\langle x, x_{0}^{\prime}-z^{\prime}\right\rangle\right| \leqq\left\langle x_{0}, x_{0}^{\prime}\right\rangle+\epsilon$; since $x_{0} \in K \cap G,\left\langle x_{0}, x_{0}^{\prime}\right\rangle<1$ by assumption; if $\epsilon$ is taken such that $\left\langle x_{0}, x_{0}^{\prime}\right\rangle+\epsilon<1$, and if $y_{0}^{\prime}=x_{0}^{\prime}-z^{\prime}$, one has $\left|\left\langle x, y_{0}^{\prime}\right\rangle\right|<1$ for every $x \in K$, which is what we set out to prove.

If $\phi(\Gamma)$ is everywhere dense in $E^{*} / H, d=0$ and the linear functional $f_{0}$ does not exist any more; ${ }^{2}$ but then, one can repeat the preceding argument for $x_{0}=0$, and this completes the proof.

2 I am indebted to the referee for calling may attention to this possibility, and to the modification the proof requires in that case. 


\section{THEOREM 3. $T$ is open for the bounded weak topology.}

We shall prove that a fundamental system of neighborhoods of the origin in $E^{*}$ for the bounded weak topology consists of the sets $K^{0}$, where $K$ is an arbitrary strongly compact subset of $E^{* *}$. Once this result is secured, the argument in Theorem 2 may be applied without change, and proves Theorem 3.

To prove our assertion, consider $E^{*}$ as a subspace of $E^{* * *}$; we have only to show that the bounded weak topology on $E^{*}$ is induced by the bounded weak* topology on $E^{* * *}$, by Theorem 1 . This will be proved if we show that any closed set $F$ in $E^{*}$ for the bounded weak topology is the intersection of $E^{*}$ with a set closed in $E^{* * *}$ for the bounded weak* topology and conversely that any such intersection is closed for the bounded weak topology. This second point is obvious, since closed sets in $E^{* * *}$ for the bounded weak* topology are defined as sets such that their intersection with any ball $S_{r}:\left\|x^{\prime \prime}\right\| \leqq r$ is weakly* closed; similarly, closed sets in $E^{*}$ for the bounded weak topology are defined as sets such that their intersection with any ball $S_{r} \cap E^{*}$ is weakly closed, and the weak topology on $S_{r} \cap E^{*}$ is induced by the weak ${ }^{*}$ topology on $S_{r}$. To prove the first point, let $F_{r}$ be the closure in $S_{r}$ of $F \cap S_{r}$ for the weak* topology; then $F \cap S_{r}$ $=F_{r} \cap\left(S_{r} \cap E^{*}\right)$, since by assumption $F \cap S_{r}$ is closed for the weak topology on $E^{*}$. Moreover, one has $F_{r^{\prime}} \cap S_{r} \cap E^{*}=F_{r} \cap S_{r} \cap E^{*}$ for every $r^{\prime}>r$; therefore, if $G$ is the union of all $F_{r}, G \cap S_{r} \cap E^{*}=F \cap S_{r}$ for every $r>0$, which proves that $F=G \cap E^{*}$. But as every $S_{r}$ is weakly* closed in $E^{* * *}, G \cap S_{r}=F_{r} \cap S_{r}$ for every $r^{\prime}>r$, and therefore $G \cap S_{r}$ is weakly* closed, which shows that $G$ is closed for the bounded weak* topology, and completes the proof.

At the end of his paper, Munroe raises the question of the closure of $T(\Delta)$ for the norm or weak topologies on $E^{*}$, when $\Delta$ is a closed (or weakly* closed) linear subspace of $E^{*}$; this, as he shows at once, is equivalent to deciding whether the sum $\Delta+\Gamma$ is or is not a closed linear subspace for these topologies. It is well known [6, p. 174] that such is not the case when $E$ is any infinite-dimensional reflexive space. This shows at the same time that statement 1 in Munroe's Theorem 3.5 is erroneous. The error in the proof comes from the tacit assumption that the sum of the unit balls in $\Delta$ and $\Gamma$ is still a neighborhood of $O$ in $\Delta+\Gamma$, which need not be the case.

\section{BiBLIOGRAPHY}

1. R. Arens, Duality in linear spaces, Duke Math. J. vol. 14 (1947) pp. 787-794.

2. S. Banach, Théorie des opérations linéaires, Warsaw, 1932. 
3. J. Dieudonné, La dualite dans les espaces rectoriels topologiques, Ann. Ecole Norm. (3) vol. 59 (1942) pp. 107-139.

4. G. Köthe, Die Quotientenrailme eines linearen vollkommenen Raumes, Math. Zeit. vol. 51 (1947) pp. 17-35.

5. M. Krein and V. Smulian, On regularly convex sets in the space conjugate to a Banach space, Ann. of Math. vol. 41 (1940) pp. 556-583.

6. G. W. Mackey, On infinite dimensional spaces, Trans. Amer. Math. Soc. vol. 57 (1945) pp. 155-207.

7. M. E. Munroe, Homomorphisms on Banach spaces, Bull. Amer. Math. Soc. vol. 54 (1948) pp. 776-781.

UNIVERSITY OF NANCY

\section{A PROOF THAT THE GROUP OF ALL HOMEO- MORPHISMS OF THE PLANE ONTO ITSELF IS LOCALLY ARCWISE CONNECTED}

M. K. FORT, JR.

Let $P$ be a plane and let $H(P)$ be the group of all homeomorphisms of the plane $P$ onto itself. We topologize $H(P)$ by defining convergence to mean uniform convergence on each compact subset of $P$. The resulting topology is equivalent to the compact-open topology defined in [1] $]^{1}$ by Fox. It is also known (see [4]) that $H(P)$ is a topological group under this topology. The result obtained in this paper is the following theorem.

Theorem. $H(P)$ is locally arcwise connected.

1. A metric for $H(P)$. We assume a rectangular coordinate system for $P$ and let $d$ be the corresponding metric for $P$. For each positive number $r$ we define $S(r)$ to be the set of all points $(u, v)$ in $P$ such that $\max (|u|,|v|) \leqq r$. If $f$ and $g$ are members of $H(P)$ we define

$$
\rho(f, g)=\sup _{r>0} \min \left(1 / r, \sup _{x \in S(r)} d(f(x), g(x))\right) .
$$

It is a routine matter to verify that $\rho$ is a distance function which defines an admissible metric for $H(P)$. A metric which is essentially the same as $\rho$ is used by M. Bebutoff in [2]. We shall make use of the fact that $\rho(f, g)<\epsilon$ if and only if $d(f(x), g(x))<\epsilon$ for all $x$ in $S(1 / \epsilon)$.

2. Isotopy and arcs. By an isotopy we shall mean a homotopy

Presented to the Society, November 27, 1948; received by the editors October 9, 1948.

${ }^{1}$ Numbers in brackets refer to the bibliography at the end of the paper. 